\title{
Fast Compressed Self-Indexes with Deterministic Linear-Time Construction ${ }^{* \dagger}$
}

\author{
J. Ian Munro', Gonzalo Navarro², and Yakov Nekrich ${ }^{3}$ \\ 1 Cheriton School of Computer Science, University of Waterloo, Canada \\ imunro@uwaterloo.ca \\ $2 \mathrm{CeBiB}$ - Center of Biotechnology and Bioengineering, Department of \\ Computer Science, University of Chile, Chile \\ gnavarro@dcc.uchile.cl \\ 3 Cheriton School of Computer Science, University of Waterloo, Canada \\ yakov.nekrich@googlemail.com
}

\begin{abstract}
We introduce a compressed suffix array representation that, on a text $T$ of length $n$ over an alphabet of size $\sigma$, can be built in $O(n)$ deterministic time, within $O(n \log \sigma)$ bits of working space, and counts the number of occurrences of any pattern $P$ in $T$ in time $O\left(|P|+\log \log _{w} \sigma\right)$ on a RAM machine of $w=\Omega(\log n)$-bit words. This new index outperforms all the other compressed indexes that can be built in linear deterministic time, and some others. The only faster indexes can be built in linear time only in expectation, or require $\Theta(n \log n)$ bits.
\end{abstract}

1998 ACM Subject Classification E.1 Data Structures, E.4 Coding and Information Theory

Keywords and phrases Succinct data structures, Self-indexes, Suffix arrays, Deterministic construction

Digital Object Identifier 10.4230/LIPIcs.ISAAC.2017.57

\section{Introduction}

The string indexing problem consists in preprocessing a string $T$ so that, later, we can efficiently find occurrences of patterns $P$ in $T$. The most popular solutions to this problem are suffix trees [29] and suffix arrays [21]. Both can be built in $O(n)$ deterministic time on a text $T$ of length $n$ over an alphabet of size $\sigma$, and the best variants can count the number of times a string $P$ appears in $T$ in time $O(|P|)$, and even in time $O\left(|P| / \log _{\sigma} n\right)$ in the word-RAM model if $P$ is given packed into $|P| / \log _{\sigma} n$ words [26]. Once counted, each occurrence can be located in $O(1)$ time. Those optimal times, however, come with two important drawbacks:

- The variants with this counting time cannot be built in $O(n)$ worst-case time.

- The data structures use $\Theta(n \log n)$ bits of space.

The reason of the first drawback is that some form of perfect hashing is always used to ensure constant time per pattern symbol (or pack of symbols). The classical suffix trees and arrays with linear-time deterministic construction offer $O(|P| \log \sigma)$ or $O(|P|+\log n)$ counting time, respectively. More recently, those times have been reduced to $O(|P|+\log \sigma)$ [10] and even to $O(|P|+\log \log \sigma)$ [14]. Simultaneously with our work, a suffix tree variant

\footnotetext{
* Funded with Basal Funds FB0001, Conicyt, Chile.

$\dagger$ The full version of this article is available at [23], https://arxiv.org/abs/1707.01743.
}

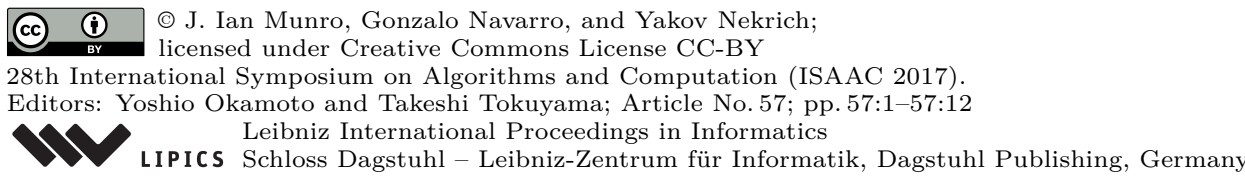


was introduced by Bille et al. [7], which can be built in linear deterministic time and counts in time $O\left(|P| / \log _{\sigma} n+\log |P|+\log \log \sigma\right)$. All those indexes, however, still suffer from the second drawback, that is, they use $\Theta(n \log n)$ bits of space. This makes them impractical in most applications that handle large text collections.

Research on the second drawback dates back to almost two decades [25], and has led to indexes using $n H_{k}(T)+o\left(n\left(H_{k}(T)+1\right)\right)$ bits, where $H_{k}(T) \leq \log \sigma$ is the $k$-th order entropy of $T$ [22], for any $k \leq \alpha \log _{\sigma} n-1$ and any constant $0<\alpha<1$. That is, the indexes use asymptotically the same space of the compressed text, and can reproduce the text and search it; thus they are called self-indexes. The fastest compressed self-indexes that can be built in linear deterministic time are able to count in time $O(|P| \log \log \sigma)[1]$ or $O\left(|P|\left(1+\log _{w} \sigma\right)\right)[6]$. There exist other compressed self-indexes that obtain times $O(|P|)[5]$ or $O\left(|P| / \log _{\sigma} n+\log _{\sigma}^{\epsilon} n\right)$ for any constant $\epsilon>0$ [18], but both rely on perfect hashing and are not built in linear deterministic time. All those compressed self-indexes use $O\left(n \frac{\log n}{b}\right)$ further bits to locate the position of each occurrence found in time $O(b)$, and to extract any substring $S$ of $T$ in time $O(|S|+b)$.

In this paper we introduce the first compressed self-index that can be built in $O(n)$ deterministic time (moreover, using $O(n \log \sigma)$ bits of space [24]) and with counting time $O\left(|P|+\log \log _{w} \sigma\right)$, where $w=\Omega(\log n)$ is the size in bits of the computer word. More precisely, we prove the following result.

- Theorem 1. On a RAM machine of $w=\Omega(\log n)$ bits, we can construct an index for a text $T$ of length $n$ over an alphabet of size $\sigma=O(n / \log n)$ in $O(n)$ deterministic time using $O(n \log \sigma)$ bits of working space. This index occupies $n H_{k}(T)+o(n \log \sigma)+O\left(n \frac{\log n}{b}\right)$ bits of space for a parameter $b$ and any $k \leq \alpha \log _{\sigma} n-1$, for any constant $0<\alpha<1$. The occurrences of a pattern string $P$ can be counted in $O\left(|P|+\log \log _{w} \sigma\right)$ time, and then each such occurrence can be located in $O(b)$ time. An arbitrary substring $S$ of $T$ can be extracted in time $O(|S|+b)$.

We obtain our results with a combination of the compressed suffix tree $\mathcal{T}$ of $T$ and the Burrows-Wheeler transform $\bar{B}$ of the reversed text $\bar{T}$. We manage to simulate the suffix tree traversal for $P$, simultaneously on $\mathcal{T}$ and on $\bar{B}$. With a combination of storing deterministic dictionaries and precomputed rank values for sampled nodes of $\mathcal{T}$, and a constant-time method to compute an extension of partial rank queries that considers small ranges in $\bar{B}$, we manage to ensure that all the suffix tree steps, except one, require constant time. The remaining one is solved with general rank queries in time $O\left(\log \log _{w} \sigma\right)$. As a byproduct, we show that the compressed sequence representations that obtain those rank times [6] can also be built in linear deterministic time.

Compared with previous work, other indexes may be faster at counting, but either they are not built in linear deterministic time $[5,18,26]$ or they are not compressed $[26,7]$. Our index outperforms all the previous compressed $[13,1,6]$, as well as some uncompressed [14], indexes that can be built deterministically.

\section{Related Work}

Let $T$ be a string of length $n$ over an alphabet of size $\sigma$ that is indexed to support searches for patterns $P$. It is generally assumed that $\sigma=o(n)$, a reasonable convention we will follow. Searches typically require to count the number of times $P$ appears in $T$, and then locate the positions of $T$ where $P$ occurs. The vast majority of the indexes for this task are suffix tree [29] or suffix array [21] variants. 
Table 1 Our results in context. The $x$ axis refers to the space used by the indexes (compressed meaning $n H_{k}(T)+o(n \log \sigma)$ bits and uncompressed meaning $\Theta(n \log n)$ bits), and the $y$ axis refers to the linear-time construction. In the cells we show the counting time for a pattern $P$. We only list the dominant alternatives, graying out those outperformed by our new results.

\begin{tabular}{|c|c|c|}
\hline & Compressed & Uncompressed \\
\hline 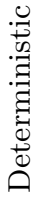 & $\begin{array}{l}|P| \log \log \sigma[1] \\
|P|\left(1+\log _{w} \sigma\right)[6] \\
|P|+\log \log _{w} \sigma \text { (ours) }\end{array}$ & $\begin{array}{l}|P|+\log \log \sigma[14] \\
|P| / \log _{\sigma} n+\log |P|+\log \log \sigma[7]\end{array}$ \\
\hline 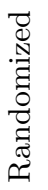 & $\begin{array}{l}|P|\left(1+\log \log _{w} \sigma\right)[6] \\
|P|[5]\end{array}$ & $|P| / \log _{\sigma} n+\log _{\sigma}^{\epsilon} n[18,26]$ \\
\hline
\end{tabular}

The suffix tree can be built in linear deterministic time [29], even on arbitrarily large integer alphabets [11]. The suffix array can be easily derived from the suffix tree in linear time, but it can also be built independently in linear deterministic time [20]. In their basic forms, these structures allow counting the number of occurrences of a pattern $P$ in $T$ in time $O(|P| \log \sigma)$ (suffix tree) or $O(|P|+\log n)$ (suffix array). Once counted, the occurrences can be located in constant time each.

Cole et al. [10] introduced the suffix trays, a simple twist on suffix trees that reduces their counting time to $O(|P|+\log \sigma)$. Fischer and Gawrychowski [14] introduced the wexponential search trees, which yield dynamic suffix trees with counting time $O(|P|+\log \log \sigma)$.

All these structures can be built in linear deterministic time, but require $\Theta(n \log n)$ bits of space, which challenges their practicality when handling large text collections.

Faster counting is possible if we resort to perfect hashing and give away the linear deterministic construction time. In the classical suffix tree, we can easily achieve $O(|P|)$ time by hashing the children of suffix tree nodes, and this is optimal in general. In the RAM model with word size $\Theta(\log n)$, and if the consecutive symbols of $P$ come packed into $|P| / \log _{\sigma} n$ words, the optimal time is instead $O\left(|P| / \log _{\sigma} n\right)$. This optimal time was recently reached by Navarro and Nekrich [26] (note that their time is not optimal if $w=\omega(\log n)$ ), with a simple application of weak-prefix search, already hinted in the original article [2]. However, even the randomized construction time of the weak-prefix search structure is $O\left(n \log ^{\epsilon} n\right)$, for any constant $\epsilon>0$. By replacing the weak-prefix search with the solution of Grossi and Vitter [18] for the last nodes of the search, and using a randomized construction of their perfect hash functions, the index of Navarro and Nekrich [26] can be built in linear randomized time and count in time $O\left(|P| / \log _{\sigma} n+\log _{\sigma}^{\epsilon} n\right)$. Only recently, simultaneously with our work, a deterministic linear-time construction algorithm was finally obtained for an index obtaining $O\left(|P| / \log _{\sigma} n+\log |P|+\log \log \sigma\right)$ counting time [7].

Still, these structures are not compressed. Compressed suffix trees and arrays appeared in the year 2000 [25]. To date, they take the space of the compressed text and replace it, in the sense that they can extract any desired substring of $T$; they are thus called self-indexes. The space occupied is measured in terms of the $k$-th order empirical entropy of $T, H_{k}(T) \leq \log \sigma$ [22], which is a lower bound on the space reached by any statistical compressor that encodes each symbol considering only the $k$ previous ones. Self-indexes may occupy as little as $n H_{k}(T)+o\left(n\left(H_{k}(T)+1\right)\right)$ bits, for any $k \leq \alpha \log _{\sigma} n-1$, for any constant $0<\alpha<1$. 
The fastest self-indexes with linear-time deterministic construction are those of Barbay et al. [1], which counts in time $O(|P| \log \log \sigma)$, and Belazzougui and Navarro [6, Thm. 7], which counts in time $O\left(|P|\left(1+\log _{w} \sigma\right)\right)$. The latter requires $O\left(n\left(1+\log _{w} \sigma\right)\right)$ construction time, but if $\log \sigma=O(\log w)$, its counting time is $O(|P|)$ and its construction time is $O(n)$.

If we admit randomized linear-time constructions, then Belazzougui and Navarro [6, Thm. 10] reach $O\left(|P|\left(1+\log \log _{w} \sigma\right)\right)$ counting time. At the expense of $O(n)$ further bits, in another work [5] they reach $O(|P|)$ counting time. Using $O(n \log \sigma)$ bits, and if $P$ comes in packed form, Grossi and Vitter [18] can count in time $O\left(|P| / \log _{\sigma} n+\log _{\sigma}^{\epsilon} n\right)$, for any constant $\epsilon>0$, however their construction requires $O(n \log \sigma)$ time.

Table 1 puts those results and our contribution in context. Our new self-index, with $O\left(|P|+\log \log _{w} \sigma\right)$ counting time, linear-time deterministic construction, and $n H_{k}(T)+$ $o(n \log \sigma)$ bits of space, dominates all the compressed indexes with linear-time deterministic construction [1, 6], as well as some uncompressed ones [14] (to be fair, we do not cover the case $\log \sigma=O(\log w)$, as in this case the previous work [6, Thm. 7] already obtains our result). Our self-index also dominates a previous one with linear-time randomized construction $[6$, Thm. 10], which we incidentally show can also be built deterministically. The only aspect in which some of the dominated indexes outperform ours is in that they may use $o\left(n\left(H_{k}(T)+1\right)\right)$ [6, Thm. 10] or $o(n)$ [6, Thm. 7] bits of redundancy, instead of our $o(n \log \sigma)$ bits.

\section{Preliminaries}

We denote by $T[i .$.$] the suffix of T[0, n-1]$ starting at position $i$ and by $T[i . . j]$ the substring that begins with $T[i]$ and ends with $T[j], T[i .]=.T[i] T[i+1] \ldots T[n-1]$ and $T[i . . j]=$ $T[i] T[i+1] \ldots T[j-1] T[j]$. We assume that the text $T$ ends with a special symbol $\$$ that lexicographically precedes all other symbols in $T$. The alphabet size is $\sigma$ and symbols are integers in $[0 . . \sigma-1]$ (so $\$$ corresponds to 0 ). In this paper, as in the previous work on this topic, we use the word RAM model of computation. A machine word consists of $w=\Omega(\log n)$ bits and we can execute standard bit and arithmetic operations in constant time. We assume for simplicity that the alphabet size $\sigma=O(n / \log n)$ (otherwise the text is almost incompressible anyway [15]). We also assume $\log \sigma=\omega(\log w)$, since otherwise our goal is already reached in previous work [6, Thm. 7].

\subsection{Rank and Select Queries}

We define three basic queries on sequences. Let $B[0 . . n-1]$ be a sequence of symbols over alphabet $[0 . . \sigma-1]$. The rank query, $\operatorname{rank}_{a}(i, B)$, counts how many times $a$ occurs among the first $i+1$ symbols in $B \operatorname{rank}_{a}(i, B)=|\{j \leq i, B[j]=a\}|$. The select query, $\operatorname{select}_{a}(i, B)$, finds the position in $B$ where $a$ occurs for the $i$-th time, $\operatorname{select}_{a}(i, B)=j$ iff $B[j]=a$ and $\operatorname{rank}_{a}(j, B)=i$. The third query is $\operatorname{access}(i, B)$, which returns simply $B[i]$.

We can answer access queries in $O(1)$ time and select queries in any $\omega(1)$ time, or vice versa, and rank queries in time $O\left(\log \log _{w} \sigma\right)$, which is optimal [6]. These structures use $n \log \sigma+o(n \log \sigma)$ bits, and we will use variants that require only compressed space. In this paper, we will show that those structures can be built in linear deterministic time.

An important special case of rank queries is the partial rank query, $\operatorname{rank}_{B[i]}(i, B)$, which asks how many times $B[i]$ occurrs in $B[0 . . i]$. Unlike general rank queries, partial rank queries can be answered in $O(1)$ time [6]. Such a structure can be built in $O(n)$ deterministic time and requires $O(n \log \log \sigma)$ bits of working and final space [24, Thm. A.4.1]. 
For this paper, we define a generalization of partial rank queries called interval rank queries, $\operatorname{rank}_{a}(i, j, B)=\left\langle\operatorname{rank}_{a}(i-1, B), \operatorname{rank}_{a}(j, B)\right\rangle$, from where in particular we can deduce the number of times $a$ occurs in $B[i . . j]$. If $a$ does not occur in $B[i . . j]$, however, this query just returns null (this is why it can be regarded as a generalized partial rank query).

In the special case where the alphabet size is small, $\log \sigma=O(\log w)$, we can represent $B$ so that rank, select, and access queries are answered in $O(1)$ time [6, Thm. 7], but we are not focusing on this case in this paper, as the problem has already been solved for this case.

\subsection{Suffix Array and Suffix Tree}

The suffix tree [29] for a string $T[0 . . n-1]$ is a compacted digital tree on the suffixes of $T$, where the leaves point to the starting positions of the suffixes. We call $X_{u}$ the string leading to suffix tree node $u$. The suffix array [21] is an array $S A[0 . . n-1]$ such that $S A[i]=j$ if and only if $T[j .$.$] is the (i+1)$-th lexicographically smallest suffix of $T$. All the occurrences of a substring $P$ in $T$ correspond to suffixes of $T$ that start with $P$. These suffixes descend from a single suffix tree node, called the locus of $P$, and also occupy a contiguous interval in the suffix array $S A$. Note that the locus of $P$ is the node $u$ closest to the root for which $P$ is a prefix of $X_{u}$. If $P$ has no locus node, then it does not occur in $T$.

\subsection{Compressed Suffix Array and Tree}

A compressed suffix array (CSA) is a compact data structure that provides the same functionality as the suffix array. The main component of a CSA is the one that allows determining, given a pattern $P$, the suffix array range $S A[i . . j]$ of the prefixes starting with $P$. Counting is then solved as $j-i+1$. For locating any cell $S A[k]$, and for extracting any substring $S$ from $T$, most CSAs make use of a sampled array $S A M_{b}$, which contains the values of $S A[i]$ such that $S A[i] \bmod b=0$ or $S A[i]=n-1$. Here $b$ is a tradeoff parameter: CSAs require $O\left(n \frac{\log n}{b}\right)$ further bits and can locate in time proportional to $b$ and extract $S$ in time proportional to $b+|S|$. We refer to a survey [25] for a more detailed description.

A compressed suffix tree [28] is formed by a compressed suffix array and other components that add up to $O(n)$ bits. These include in particular a representation of the tree topology that supports constant-time computation of the preorder of a node, its number of children, its $j$-th child, its number of descendant leaves, and lowest common ancestors, among others [27]. Computing node preorders is useful to associate satellite information to the nodes.

Both the compressed suffix array and tree can be built in $O(n)$ deterministic time using $O(n \log \sigma)$ bits of space [24].

\subsection{Burrows-Wheeler Transform and FM-index}

The Burrows-Wheeler Transform (BWT) [8] of a string $T[0 . . n-1]$ is another string $B[0 . . n-1]$ obtained by sorting all possible rotations of $T$ and writing the last symbol of every rotation (in sorted order). The BWT is related to the suffix array by the identity $B[i]=T[(S A[i]-1)$ $\bmod n]$. Hence, we can build the BWT by sorting the suffixes and writing the symbols that precede the suffixes in lexicographical order.

The FM-index $[12,13]$ is a CSA that builds on the BWT. It consists of the following three main components: (1) the BWT $B$ of $T$; $(2)$ the array $A c c[0 . . \sigma-1]$ where $A c c[i]$ holds the total number of symbols $a<i$ in $T$ (or equivalently, the total number of symbols $a<i$ in $B) ;(3)$ the sampled array $S A M_{b}$. 
The interval of a pattern string $P[0 . . m-1]$ in the suffix array $S A$ can be computed on the BWT $B$. The interval is computed backwards: for $i=m-1, m-2, \ldots$, we identify the interval of $P[i . . m-1]$ in $B$. The interval is initially the whole $B[0 . . n-1]$. Suppose that we know the interval $B\left[i_{1} . . j_{1}\right]$ that corresponds to $P[i+1 . . m-1]$. Then the interval $B\left[i_{2} . . j_{2}\right]$ that corresponds to $P[i . . m-1]$ is computed as $i_{2}=A c c[a]+\operatorname{rank}_{c}\left(i_{1}-1, B\right)$ and $j_{2}=A c c[a]+\operatorname{rank}_{c}\left(j_{1}, B\right)-1$, where $a=P[i]$. Thus the interval of $P$ is found by answering $2 m$ rank queries. Any sequence representation offering rank and access queries can then be applied on $B$ to obtain an FM-index.

An important procedure on the FM-index is the computation of the function $L F$, defined as: if $S A[j]=i+1$, then $S A[L F(j)]=i$. $L F$ can be computed with access and partial rank queries on $B, L F(j)=\operatorname{rank}_{B[j]}(i, B)+A c c[B[j]]-1$, and thus constant-time computation of $L F$ is possible. Using $S A M_{b}$ and $O(b)$ applications of $L F$, we can locate any cell $S A[r]$. A similar procedure extracts any substring $S$ of $T$ with $O(b+|S|)$ applications of $L F$.

\section{$4 \quad$ Small Interval Rank Queries}

We start by showing how a compressed data structure that supports select queries can be extended to support a new kind of queries that we dub small interval rank queries. An interval query $\operatorname{rank}_{a}(i, j, B)$ is a small interval rank query if $j-i \leq \log ^{2} \sigma$. Our compressed index relies on the following result.

- Lemma 2. Suppose that we are given a data structure that supports access queries on a sequence $C[0 . . m-1]$, on alphabet $[0 . . \sigma-1]$, in time $t$. Then, using $O(m \log \log \sigma)$ additional bits, we can support small interval rank queries on $C$ in $O(t)$ time.

Proof. We split $C$ into groups $G_{i}$ of $\log ^{2} \sigma$ consecutive symbols, $G_{i}=C\left[i \log ^{2} \sigma . .(i+\right.$ 1) $\left.\log ^{2} \sigma-1\right]$. Let $A_{i}$ denote the sequence of the distinct symbols that occur in $G_{i}$. Storing $A_{i}$ directly would need $\log \sigma$ bits per symbol. Instead, we encode each element of $A_{i}$ as its first position in $G_{i}$, which needs only $O(\log \log \sigma)$ bits. With this encoded sequence, since we have $O(t)$-time access to $C$, we have access to any element of $A_{i}$ in time $O(t)$. In addition, we store a succinct SB-tree [17] on the elements of $A_{i}$. This structure uses $O(p \log \log u)$ bits to index $p$ elements in $[1 . . u]$, and supports predecessor (and membership) queries in time $O(\log p / \log \log u)$ plus one access to $A_{i}$. Since $u=\sigma$ and $p \leq \log ^{2} \sigma$, the query time is $O(t)$ and the space usage is bounded by $O(m \log \log \sigma)$ bits.

For each $a \in A_{i}$ we also keep the increasing list $I_{a, i}$ of all the positions where $a$ occurs in $G_{i}$. Positions are stored as differences with the left border of $G_{i}$ : if $C[j]=a$, we store the difference $j-i \log ^{2} \sigma$. Hence elements of $I_{a, i}$ can also be stored in $O(\log \log \sigma)$ bits per symbol, adding up to $O(m \log \log \sigma)$ bits. We also build an SB-tree on top of each $I_{a, i}$ to provide for predecessor searches.

Using the SB-trees on $A_{i}$ and $I_{a, i}$, we can answer small interval rank queries $\operatorname{rank}_{a}(x, y, C)$. Consider a group $G_{i}=C\left[i \log ^{2} \sigma . .(i+1) \log ^{2} \sigma-1\right]$, an index $k$ such that $i \log ^{2} \sigma \leq k \leq$ $(i+1) \log ^{2} \sigma$, and a symbol $a$. We can find the largest $i \log ^{2} \sigma \leq r \leq k$ such that $C[r]=a$, or determine it does not exist: First we look for the symbol $a$ in $A_{i}$; if $a \in A_{i}$, we find the predecessor of $k-i \log ^{2} \sigma$ in $I_{a, i}$.

Now consider an interval $C[x . . y]$ of size at most $\log ^{2} \sigma$. It intersects at most two groups, $G_{i}$ and $G_{i-1}$. We find the rightmost occurrence of symbol $a$ in $C[x . . y]$ as follows. First we look for the rightmost occurrence $y^{\prime} \leq y$ of $a$ in $G_{i}$; if $a$ does not occur in $C\left[i \log ^{2} \sigma . . y\right]$, we look for the rightmost occurrence $y^{\prime} \leq i \log ^{2} \sigma-1$ of $a$ in $G_{i-1}$. If this is $\geq x$, we find the leftmost occurrence $x^{\prime}$ of $a$ in $C[x . . y]$ using a symmetric procedure. When $x^{\prime} \leq y^{\prime}$ are 
found, we can compute $\operatorname{rank}_{a}\left(x^{\prime}, C\right)$ and $\operatorname{rank}_{a}\left(y^{\prime}, C\right)$ in $O(1)$ time by answering partial rank queries (Section 3.1). These are supported in $O(1)$ time and $O(m \log \log \sigma)$ bits. The answer is then $\left\langle\operatorname{rank}_{a}\left(x^{\prime}, C\right)-1, \operatorname{rank}_{a}\left(y^{\prime}, C\right)\right\rangle$, or null if $a$ does not occur in $C[x . . y]$.

The construction of the small interval rank data structure is dominated by the time needed to build the succinct SB-trees [17]. These are simply B-trees with arity $O(\sqrt{\log u})$ and height $O(\log p / \log \log u)$, where in each node a Patricia tree for $O(\log \log u)$-bit chunks of the keys are stored. To build the structure in $O(\log p / \log \log u)$ time per key, we only need to build those Patricia trees in linear time. Given that the total number of bits of all the keys to insert in a Patricia tree is $O(\sqrt{\log u} \log \log u)$, we do not even need to build the Patricia tree. Instead, a universal precomputed table may answer any Patricia tree search for any possible set of keys and any possible pattern, in constant time. The size of the table is $O\left(2^{O(\sqrt{\log u} \log \log u)} \sqrt{\log u}\right)=o(u)$ bits (the authors [17] actually use a similar table to answer queries). For our values of $p$ and $u$, the construction requires $O(m t)$ time and the universal table is of $o(\sigma)$ bits.

\section{Compressed Index}

We classify the nodes of the suffix tree $\mathcal{T}$ of $T$ into heavy, light, and special, as in previous work $[26,24]$. Let $d=\log \sigma$. A node $u$ of $\mathcal{T}$ is heavy if it has at least $d$ leaf descendants and light otherwise. We say that a heavy node $u$ is special if it has at least two heavy children.

For every special node $u$, we construct a deterministic dictionary [19] $D_{u}$ that contains the labels of all the heavy children of $u$ : If the $j$ th child of $u, u_{j}$, is heavy and the first symbol on the edge from to $u$ to $u_{j}$ is $a_{j}$, then we store the key $a_{j}$ in $D_{u}$ with $j$ as satellite data. If a heavy node $u$ has only one heavy child $u_{j}$ and $d$ or more light children, then we also store the data structure $D_{u}$ (containing only that heavy child of $u$ ). If, instead, a heavy node has one heavy child and less than $d$ light children, we just keep the index of the heavy child using $O(\log d)=O(\log \log \sigma)$ bits.

The second component of our index is the Burrows-Wheeler Transform $\bar{B}$ of the reverse text $\bar{T}$. We store a data structure that supports rank, partial rank, select, and access queries on $\bar{B}$. It is sufficient for us to support access and partial rank queries in $O(1)$ time and rank queries in $O\left(\log \log _{w} \sigma\right)$ time. We also construct the data structure described in Lemma 2, which supports small interval rank queries in $O(1)$ time. Finally, we explicitly store the answers to some rank queries. Let $\bar{B}\left[l_{u} . . r_{u}\right]$ denote the range of $\overline{X_{u}}$, where $\overline{X_{u}}$ is the reverse of $X_{u}$, for a suffix tree node $u$. For all data structures $D_{u}$ and for every symbol $a \in D_{u}$ we store the values of $\operatorname{rank}_{a}\left(l_{u}-1, \bar{B}\right)$ and $\operatorname{rank}_{a}\left(r_{u}, \bar{B}\right)$.

Let us show how to store the selected precomputed answers to rank queries in $O(\log \sigma)$ bits per query. Following a known scheme [16], we divide the sequence $\bar{B}$ into chunks of size $\sigma$. For each symbol $a$, we encode the number $d_{k}$ of times $a$ occurs in each chunk $k$ in a binary sequence $A_{a}=01^{d_{0}} 01^{d_{1}} 01^{d_{2}} \ldots$. If a symbol $\bar{B}[i]$ belongs to chunk $k=\lfloor i / \sigma\rfloor$, then $\operatorname{rank}_{a}(i, \bar{B})$ is $\operatorname{select}_{0}\left(k+1, A_{a}\right)-k$ plus the number of times $a$ occurs in $\bar{B}[k \sigma . . i]$. The former value is computed in $O(1)$ time with a structure that uses $\left|A_{a}\right|+o\left(\left|A_{a}\right|\right)$ bits [9], whereas the latter value is in $[0, \sigma]$ and thus can be stored in $D_{u}$ using just $O(\log \sigma)$ bits. The total size of all the sequences $A_{a}$ is $O(n)$ bits.

Therefore, $D_{u}$ needs $O(\log \sigma)$ bits per element. The total number of elements in all the structures $D_{u}$ is equal to the number of special nodes plus the number of heavy nodes with one heavy child and at least $d$ light children. Hence all $D_{u}$ contain $O(n / d)$ symbols and use $O((n / d) \log \sigma)=O(n)$ bits of space. Indexes of heavy children for nodes with only one heavy child and less than $d$ light children add up to $O(n \log \log \sigma)$ bits. The structures for partial rank and small interval rank queries on $\bar{B}$ use $O(n \log \log \sigma)$ further bits. Since we assume that $\sigma$ is $\omega(1)$, we can simplify $O(n \log \log \sigma)=o(n \log \sigma)$. 
The sequence representation that supports access and rank queries on $\bar{B}$ can be made to use $n H_{k}(T)+o\left(n\left(H_{k}(T)+1\right)\right)$ bits, by exploiting the fact that it is built on a BWT [6, Thm. 10]. ${ }^{1}$ We note that they use constant-time select queries on $\bar{B}$ instead of constant-time access, so they can use select queries to perform $L F^{-1}$-steps in constant time. Instead, with our partial rank queries, we can perform $L F$-steps in constant time (recall Section 3.4), and thus have constant-time access instead of constant-time select on $\bar{B}$ (we actually do not use query select at all). They avoid this solution because partial rank queries require $o(n \log \sigma)$ bits, which can be more than $o\left(n\left(H_{k}(T)+1\right)\right)$, but we are already paying this price.

Apart from this space, array $A c c$ needs $O(\sigma \log n)=O(n)$ bits and $S A M_{b}$ uses $O\left(n \frac{\log n}{b}\right)$. The total space usage of our self-index then adds up to $n H_{k}(T)+o(n \log \sigma)+O\left(n \frac{\log n}{b}\right)$ bits.

\section{Pattern Search}

Given a query string $P$, we will find in time $O\left(|P|+\log \log _{w} \sigma\right)$ the range of the reversed string $\bar{P}$ in $\bar{B}$. A backward search for $P$ in $B$ will be replaced by an analogous backward search for $\bar{P}$ in $\bar{B}$, that is, we will find the range of $\overline{P[0 . . i]}$ if the range of $\overline{P[0 . . i-1]}$ is known. Let $\left[l_{i} . . r_{i}\right]$ be the range of $\overline{P[0 . . i]}$. We can compute $l_{i}$ and $r_{i}$ from $l_{i-1}$ and $r_{i-1}$ as $l_{i}=A c c[a]+\operatorname{rank}_{a}\left(l_{i-1}-1, \bar{B}\right)$ and $r_{i}=A c c[a]+\operatorname{rank}_{a}\left(r_{i-1}, \bar{B}\right)-1$, for $a=P[i]$. Using our auxiliary data structures on $\bar{B}$ and the additional information stored in the nodes of the suffix tree $\mathcal{T}$, we can answer the necessary rank queries in constant time (with one exception). The idea is to traverse the suffix tree $\mathcal{T}$ in synchronization with the forward search on $\bar{B}$, until the locus of $P$ is found or we determine that $P$ does not occur in $T$.

Our procedure starts at the root node of $\mathcal{T}$, with $l_{-1}=0, r_{-1}=n-1$, and $i=0$. We compute the ranges $\bar{B}\left[l_{i} . . r_{i}\right]$ that correspond to $\overline{P[0 . . i]}$ for $i=0, \ldots,|P|-1$. Simultaneously, we move down in the suffix tree. Let $u$ denote the last visited node of $\mathcal{T}$ and let $a=P[i]$. We denote by $u_{a}$ the next node that we must visit in the suffix tree, i.e., $u_{a}$ is the locus of $P[0 . . i]$. We can compute $l_{i}$ and $r_{i}$ in $O(1)$ time if $\operatorname{rank}_{a}\left(r_{i-1}, \bar{B}\right)$ and $\operatorname{rank}_{a}\left(l_{i-1}-1, \bar{B}\right)$ are known. We will show below that these queries can be answered in constant time because either (a) the answers to rank queries are explicitly stored in $D_{u}$ or (b) the rank query that must be answered is a small interval rank query. The only exception is the situation when we move from a heavy node to a light node in the suffix tree; in this case the rank query takes $O\left(\log \log _{w} \sigma\right)$ time. We note that, once we are in a light node, we need not descend in $\mathcal{T}$ anymore; it is sufficient to maintain the interval in $\bar{B}$.

For ease of description we distinguish between the following cases.

1. Node $u$ is heavy and $a \in D_{u}$. In this case we identify the heavy child $u_{a}$ of $u$ that is labeled with $a$ in constant time using the deterministic dictionary. We can also find $l_{i}$ and $r_{i}$ in time $O(1)$ because $\operatorname{rank}_{a}\left(l_{i-1}-1, \bar{B}\right)$ and $\operatorname{rank}_{a}\left(r_{i-1}, \bar{B}\right)$ are stored in $D_{u}$.

2. Node $u$ is heavy and $a \notin D_{u}$. In this case $u_{a}$, if it exists, is a light node. We then find it with two standard rank queries on $\bar{B}$, in order to compute $l_{i}$ and $r_{i}$ or determine that $P$ does not occur in $T$.

3. Node $u$ is heavy but we do not keep a dictionary $D_{u}$ for the node $u$. In this case $u$ has at most one heavy child and less than $d$ light children. We have two subcases:

a. If $u_{a}$ is the (only) heavy node, we find this out with a single comparison, as the heavy node is identified in $u$. However, the values $\operatorname{rank}_{a}\left(l_{i-1}-1, \bar{B}\right)$ and $\operatorname{rank}_{a}\left(r_{i-1}, \bar{B}\right)$ are not stored in $u$. To compute them, we exploit the fact that the number of non- $a$ 's in $\bar{B}\left[l_{i-1} . . r_{i-1}\right]$ is less than $d^{2}$, as all the children apart from $u_{a}$ are light and less than $d$.

${ }^{1}$ In fact it is $n H_{k}(\bar{T})$, but this is $n H_{k}(T)+O(\log n)$ [12, Thm. A.3]. 
Therefore, the first and the last occurrences of $a$ in $\bar{B}\left[l_{i-1} . . r_{i-1}\right]$ must be at distance less than $d^{2}$ from the extremes $l_{i-1}$ and $r_{i-1}$, respectively. Therefore, a small interval rank query, $\operatorname{rank}_{a}\left(l_{i-1}, l_{i-1}+d^{2}, \bar{B}\right)$, gives us $\operatorname{rank}_{a}\left(l_{i-1}-1, \bar{B}\right)$, since there is for sure an $a$ in the range. Analogously, $\operatorname{rank}_{a}\left(r_{i-1}-d^{2}, r_{i-1}, \bar{B}\right)$ gives $\operatorname{us}^{\operatorname{rank}_{a}}\left(r_{i-1}, \bar{B}\right)$.

b. If $u_{a}$ is a light node, we compute $l_{i}$ and $r_{i}$ with two standard rank queries on $\bar{B}$ (or we might determine that $P$ does not appear in $T$ ).

4. Node $u$ is light. In this case, $P[0 . . i-1]$ occurs at most $d$ times in $T$. Hence $\overline{P[0 . . i-1]}$ also occurs at most $d$ times in $\bar{T}$ and $r_{i-1}-l_{i-1} \leq d$. Therefore we can compute $r_{i}$ and $l_{i}$ in $O(1)$ time by answering a small interval rank query, $\left\langle\operatorname{rank}_{a}\left(l_{i-1}-1, \bar{B}\right), \operatorname{rank}_{a}\left(r_{i-1}, \bar{B}\right)\right\rangle$. If this returns null, then $P$ does not occur in $T$.

5. We are on an edge of the suffix tree between a node $u$ and some child $u_{j}$ of $u$. In this case all the occurrences of $P[0 . . i-1]$ in $T$ are followed by the same symbol, $c$, and all the occurrences of $\overline{P[0 . . i-1]}$ are preceded by $c$ in $\bar{T}$. Therefore $\bar{B}\left[l_{i-1} . . r_{i-1}\right]$ contains only the symbol $c$. This situation can be verified with access and partial rank queries on $\bar{B}: \bar{B}\left[r_{i-1}\right]=\bar{B}\left[l_{i-1}\right]=c$ and $\operatorname{rank}_{c}\left(r_{i-1}, \bar{B}\right)-\operatorname{rank}_{c}\left(l_{i-1}, \bar{B}\right)=r_{i-1}-l_{i-1}$. In this case, if $a \neq c$, then $P$ does not occur in $T$; otherwise we obtain the new range with the partial rank query $\operatorname{rank}_{c}\left(r_{i-1}, \bar{B}\right)$, and $\operatorname{rank}_{c}\left(l_{i-1}-1, \bar{B}\right)=\operatorname{rank}_{c}\left(r_{i-1}, \bar{B}\right)-\left(r_{i-1}-l_{i-1}+1\right)$. Note that if $u$ is light we do not need to consider this case; we may directly apply case 4 .

Except for the cases 2 and $3 \mathrm{~b}$, we can find $l_{i}$ and $r_{i}$ in $O(1)$ time. In cases 2 and $3 \mathrm{~b}$ we need $O\left(\log \log _{w} \sigma\right)$ time to answer general rank queries. However, these cases only take place when the node $u$ is heavy and its child $u_{a}$ is light. Since all descendants of a light node are light, those cases occur only once along the traversal of $P$. Hence the total time to find the range of $\bar{P}$ in $\bar{B}$ is $O\left(|P|+\log \log _{w} \sigma\right)$. Once the range is known, we can count and report all occurrences of $\bar{P}$ in the standard way.

\section{Linear-Time Construction}

\subsection{Sequences and Related Structures}

Apart from constructing the BWT $\bar{B}$ of $\bar{T}$, which is a component of the final structure, the linear-time construction of the other components requires that we also build, as intermediate structures, the BWT $B$ of $T$, and the compressed suffix trees $\overline{\mathcal{T}}$ and $\mathcal{T}$ of $\bar{T}$ and $T$, respectively. All these are built in $O(n)$ deterministic time and using $O(n \log \sigma)$ bits of space [24]. We also keep, on top of both $\bar{B}$ and $B, O(n \log \log \sigma)$-bit data structures able to report, for any interval $\bar{B}[i . . j]$ or $B[i . . j]$, all the distinct symbols from this interval, and their frequencies in the interval. The symbols are retrieved in arbitrary order. These auxiliary data structures can also be constructed in $O(n)$ time [24, Sec. A.5]. On top of the sequences $B$ and $\bar{B}$, we build the representation that supports access in $O(1)$ and rank in $O\left(\log \log _{w} \sigma\right)$ time [6]. This was built using perfect hashing, but it can also be built deterministically [4, Lem. 11].

\subsection{Structures $D_{u}$}

The most complex part of the construction is to fill the data of the $D_{u}$ structures. We visit all the nodes of $\mathcal{T}$ and identify those nodes $u$ for which the data structure $D_{u}$ must be constructed. This can be easily done in linear time, by using the constant-time computation of the number of descendant leaves. To determine if we must build $D_{u}$, we traverse its children $u_{1}, u_{2}, \ldots$ and count their descendant leaves to decide if they are heavy or light.

We use a bit vector $D$ to mark the preorders of the nodes $u$ for which $D_{u}$ will be constructed: If $p$ is the preorder of node $u$, then it stores a structure $D_{u}$ iff $D[p]=1$, in which case $D_{u}$ is stored in an array at position $\operatorname{rank}_{1}(D, p)$. If, instead, $u$ does not store $D_{u}$ 
but it has one heavy child, we store its child rank in another array indexed by $\operatorname{rank}_{0}(D, p)$, using $\log \log \sigma$ bits per cell.

The main difficulty is how to compute the symbols $a$ to be stored in $D_{u}$, and the ranges $\bar{B}\left[l_{u}, r_{u}\right]$, for all the selected nodes $u$. It is not easy to do this through a preorder traversal of $\mathcal{T}$ because we would need to traverse edges that represent many symbols. Our approach, instead, is inspired by the navigation of the suffix-link tree using two BWTs given by Belazzougui et al. [3]. Let $\mathcal{T}_{w}$ denote the tree whose edges correspond to Weiner links between internal nodes in $\mathcal{T}$. That is, the root of $\mathcal{T}_{w}$ is the same root of $\mathcal{T}$ and, if we have internal nodes $u, v \in \mathcal{T}$ where $X_{v}=a \cdot X_{u}$ for some symbol $a$, then $v$ descends from $u$ by the symbol $a$ in $\mathcal{T}_{w}$. It is well known that that the nodes of $\mathcal{T}_{w}$ are the internal nodes of $\mathcal{T}$.

We do not build $\mathcal{T}_{w}$ explicitly, but just traverse its nodes conceptually in depth-first order and compute the symbols to store in the structures $D_{u}$ and the intervals in $\bar{B}$. Let $u$ be the current node of $\mathcal{T}$ in this traversal and $\bar{u}$ its corresponding locus in $\overline{\mathcal{T}}$. Assume for now that $\bar{u}$ is a node, too. Let $\left[l_{u}, r_{u}\right]$ be the interval of $X_{u}$ in $B$ and $\left[l_{\bar{u}}, r_{\bar{u}}\right]$ be the interval of the reverse string $X_{\bar{u}}$ in $\bar{B} .^{2}$ Our algorithm starts at the root nodes of $\mathcal{T}_{w}, \mathcal{T}$, and $\overline{\mathcal{T}}$, which correspond to the empty string, and the intervals in $B$ and $\bar{B}$ are $\left[l_{u}, r_{u}\right]=\left[l_{\bar{u}}, r_{\bar{u}}\right]=[0, n-1]$. We will traverse only the heavy nodes, yet in some cases we will have to work on all the nodes. We ensure that on heavy nodes we work at most $O(\log \sigma)$ time, and at most $O(1)$ time on arbitrary nodes.

Upon arriving at each node $u$, we first compute its heavy children. From the topology of $\mathcal{T}$ we identify the interval $\left[l_{i}, r_{i}\right]$ for every child $u_{i}$ of $u$, by counting leaves in the subtrees of the successive children of $u$. By reporting all the distinct symbols in $\bar{B}\left[l_{\bar{u}} . r_{\bar{u}}\right]$ with their frequencies, we identify the labels of those children. However, the labels are retrieved in arbitrary order and we cannot afford sorting them all. Yet, since the labels are associated with their frequencies in $\bar{B}\left[l_{\bar{u}} . . r_{\bar{u}}\right]$, which match their number of leaves in the subtrees of $u$, we can discard the labels of the light children, that is, those appearing less than $d$ times in $\bar{B}\left[l_{\bar{u}} . . r_{\bar{u}}\right]$. The remaining, heavy, children are then sorted and associated with the successive heavy children $u_{i}$ of $u$ in $\mathcal{T}$.

If our preliminary pass marked that a $D_{u}$ structure must be built, we construct at this moment the deterministic dictionary [19] with the labels $a$ of the heavy children of $u$ we have just identified, and associate them with the satellite data $\operatorname{rank}_{a}\left(l_{\bar{u}}-1, \bar{B}\right)$ and $\operatorname{rank}_{a}\left(r_{\bar{u}}, \bar{B}\right)$. This construction takes $O(\log \sigma)$ time per element, but it includes only heavy nodes.

We now find all the Weiner links from $u$. For every (heavy or light) child $u_{i}$ of $u$, we compute the list $L_{i}$ of all the distinct symbols that occur in $B\left[l_{i} . . r_{i}\right]$. We mark those symbols $a$ in an array $V[0 . . \sigma-1]$ that holds three possible values: not seen, seen, and seen (at least) twice. If $V[a]$ is not seen, then we mark it as seen; if it is seen, we mark it as seen twice; otherwise we leave it as seen twice. We collect a list $E_{u}$ of the symbols that are seen twice along this process, in arbitrary order. For every symbol $a$ in $E_{u}$, there is an Weiner link from $u$ labeled by $a$ : Let $X=X_{u}$; if $a$ occurred in $L_{i}$ and $L_{j}$ then both $a X a_{i}$ and $a X a_{j}$ occur in $T$ and there is a suffix tree node that corresponds to the string $a X$. The total time to build $E_{u}$ amortizes to $O(n)$ : for each child $v$ of $u$, we pay $O(1)$ time for each child the node $\bar{v}$ has in $\overline{\mathcal{T}}$; each node in $\overline{\mathcal{T}}$ contributes once to the cost.

The targets of the Weiner links from $u$ in $\mathcal{T}$ correspond to the children of the node $\bar{u}$ in $\overline{\mathcal{T}}$. To find them, we collect all the distinct symbols in $B\left[l_{u} . . r_{u}\right]$ and their frequencies. Again, we discard the symbols with frequency less than $d$, as they will lead to light nodes, which we do not have to traverse. The others are sorted and associated with the successive heavy

2 In the rest of the paper we wrote $\bar{B}\left[l_{u} . . r_{u}\right]$ instead of $\bar{B}\left[l_{\bar{u}} . . r_{\bar{u}}\right]$ for simplicity, but this may cause confusion in this section. 
children of $\bar{u}$. By counting leaves in the successive children, we obtain the intervals $\bar{B}\left[l_{i}^{\prime} . . r_{i}^{\prime}\right]$ corresponding to the heavy children $\bar{u}_{i}^{\prime}$ of $\bar{u}$.

We are now ready to continue the traversal of $\mathcal{T}_{w}$ : for each Weiner link from $u$ by symbol $a$ leading to a heavy node, which turns out to be the $i$-th child of $\bar{u}$, we know that its node in $\overline{\mathcal{T}}$ is $\bar{u}_{i}^{\prime}$ (computed from $\bar{u}$ using the tree topology) and its interval is $\bar{B}\left[l_{i}^{\prime} . . r_{i}^{\prime}\right]$. To compute the corresponding interval on $B$, we use the backward step operation, $B[x, y]=B\left[A c c[a]+\operatorname{rank}_{a}\left(l_{u}-1, B\right), A c c[a]+\operatorname{rank}_{a}\left(r_{u}, B\right)-1\right]$. This requires $O\left(\log \log _{w} \sigma\right)$ time, but applies only to heavy nodes. Finally, the corresponding node in $\mathcal{T}$ is obtained in constant time as the lowest common ancestor of the $x$-th and the $y$-th leaves of $\mathcal{T}$.

In the description above we assumed for simplicity that $\bar{u}$ is a node in $\overline{\mathcal{T}}$. In the general case $\bar{u}$ can be located on an edge of $\overline{\mathcal{T}}$. This situation arises when all occurrences of $\overline{X_{u}}$ in the reverse text $\bar{T}$ are followed by the same symbol $a$. In this case there is at most one Weiner link from $u$; the interval in $\bar{B}$ does not change as we follow that link.

A recursive traversal of $\mathcal{T}_{w}$ might require $O(n \sigma \log n)$ bits for the stack, because we store several integers associated to heavy children during the computation of each node $u$. We can reduce this to $O\left(\sigma \log ^{2} n\right)=O(n \log \sigma)$ by standard means [3, Lem. 1].

We have spent at most $O(\log \sigma)$ time on heavy nodes, which are $O(n / d)=O(n / \log \sigma)$ in total, thus these costs add up to $O(n)$. All other costs that apply to arbitrary nodes are $O(1)$. The structures for partial rank queries (and the succinct SB-trees) can also be built in linear deterministic time, as seen in Section 4. Then our index is constructed in $O(n)$ time.

\section{Conclusions}

We have shown how to build, in $O(n)$ deterministic time and using $O(n \log \sigma)$ bits of working space, a compressed self-index for a text $T$ of length $n$ over an alphabet of size $\sigma$ that searches for patterns $P$ in time $O\left(|P|+\log \log _{w} \sigma\right)$, on a $w$-bit word RAM machine. This improves upon previous compressed self-indexes requiring $O(|P| \log \log \sigma)[1]$ or $O\left(|P|\left(1+\log _{w} \sigma\right)\right)$ [6] time, on previous uncompressed indexes requiring $O(|P|+\log \log \sigma$ ) time [14] (but that supports dynamism), and on previous compressed self-indexes requiring $O\left(|P|\left(1+\log \log _{w} \sigma\right)\right)$ time and randomized construction (which we now showed how to build in linear deterministic time) [6]. The only indexes offering better search time require randomized construction $[5,18,26]$ or $\Theta(n \log n)$ bits of space $[26,7]$.

In our extended paper [23], we show that using $O(n \log \sigma)$ bits of space, we can build in $O(n)$ deterministic time an index that searches in time $O\left(|P| / \log _{\sigma} n+\log n(\log \log n)^{2}\right)$. Current indexes obtaining similar counting time require $O(n \log \sigma)$ construction time [18] or higher [26], or $O(n \log n)$ bits of space [26, 7].

It is not clear if $O(|P|)$ time, or even $O\left(|P| / \log _{\sigma} n\right)$, query time can be achieved with a linear deterministic construction time, even if we allow $O(n \log n)$ bits of space for the index (this was recently approached, but some additive polylog factors remain [7]). This is the most interesting open problem for future research.

\section{References}

1 J. Barbay, F. Claude, T. Gagie, G. Navarro, and Y. Nekrich. Efficient fully-compressed sequence representations. Algorithmica, 69(1):232-268, 2014.

2 D. Belazzougui, P. Boldi, R. Pagh, and S. Vigna. Fast prefix search in little space, with applications. In Proc. 18th ESA, LNCS 6346, pages 427-438, 2010.

3 D. Belazzougui, F. Cunial, J. Kärkkäinen, and V. Mäkinen. Versatile succinct representations of the bidirectional Burrows-Wheeler transform. In Proc. 21st ESA, pages 133-144, 2013. 
4 D. Belazzougui, F. Cunial, J. Kärkkäinen, and V. Mäkinen. Linear-time string indexing and analysis in small space. CoRR, abs/1609.06378, 2016.

5 D. Belazzougui and G. Navarro. Alphabet-independent compressed text indexing. ACM Trans. Alg., 10(4):article 23, 2014.

6 D. Belazzougui and G. Navarro. Optimal lower and upper bounds for representing sequences. ACM Trans. Alg., 11(4):article 31, 2015.

7 P. Bille, I. L. Gørtz, and F. R. Skjoldjensen. Deterministic indexing for packed strings. In Proc. 28th CPM, LIPIcs 78, page article 6, 2017.

8 M. Burrows and D. Wheeler. A block sorting lossless data compression algorithm. Technical Report 124, Digital Equipment Corporation, 1994.

9 D. R. Clark. Compact PAT Trees. PhD thesis, University of Waterloo, Canada, 1996.

10 R. Cole, T. Kopelowitz, and M. Lewenstein. Suffix trays and suffix trists: Structures for faster text indexing. Algorithmica, 72(2):450-466, 2015.

11 M. Farach. Optimal suffix tree construction with large alphabets. In Proc. 38th FOCS, pages 137-143, 1997.

12 P. Ferragina and G. Manzini. Indexing compressed text. J. ACM, 52(4):552-581, 2005.

13 P. Ferragina, G. Manzini, V. Mäkinen, and G. Navarro. Compressed representations of sequences and full-text indexes. ACM Trans. Alg., 3(2):article 20, 2007.

14 J. Fischer and P. Gawrychowski. Alphabet-dependent string searching with wexponential search trees. In Proc. 26th CPM, LNCS 9133, pages 160-171, 2015.

15 T. Gagie. Large alphabets and incompressibility. Inf. Proc. Lett., 99(6):246-251, 2006.

16 A. Golynski, J. I. Munro, and S. S. Rao. Rank/select operations on large alphabets: a tool for text indexing. In Proc. 17th SODA, pages 368-373, 2006.

17 R. Grossi, A. Orlandi, R. Raman, and S. S. Rao. More haste, less waste: Lowering the redundancy in fully indexable dictionaries. In Proc. 26th STACS, pages 517-528, 2009.

18 R. Grossi and J. S. Vitter. Compressed suffix arrays and suffix trees with applications to text indexing and string matching. SIAM J. Comp., 35(2):378-407, 2005.

19 T. Hagerup, P. Bro Miltersen, and R. Pagh. Deterministic dictionaries. J. Alg., 41(1):69 85, 2001.

20 J. Kärkkäinen, P. Sanders, and S. Burkhardt. Linear work suffix array construction. J. ACM, 53(6):918-936, 2006.

21 U. Manber and G. Myers. Suffix arrays: a new method for on-line string searches. SIAM J. Comp., 22(5):935-948, 1993.

22 G. Manzini. An analysis of the Burrows-Wheeler transform. J. ACM, 48(3):407-430, 2001.

23 J. I. Munro, G. Navarro, and Y. Nekrich. Fast compressed self-indexes with deterministic linear-time construction. CoRR, abs/1707.01743, 2017.

24 J. I. Munro, G. Navarro, and Y. Nekrich. Space-efficient construction of compressed indexes in deterministic linear time. In Proc. 28th SODA, pages 408-424, 2017.

25 G. Navarro and V. Mäkinen. Compressed full-text indexes. ACM Comp. Surv., 39(1):article $2,2007$.

26 G. Navarro and Y. Nekrich. Time-optimal top- $k$ document retrieval. SIAM J. Comp., 46(1):89-113, 2017.

27 G. Navarro and K. Sadakane. Fully-functional static and dynamic succinct trees. ACM Trans. Alg., 10(3):article 16, 2014.

28 K. Sadakane. Compressed suffix trees with full functionality. Theor. Comp. Sys., 41(4):589607, 2007.

29 P. Weiner. Linear pattern matching algorithms. In Proc. 14th FOCS, pages 1-11, 1973. 